\title{
Time-Selective Signaling and Reception for Communication over Multipath Fading Channels
}

\author{
Srikrishna Bhashyam, Student Member, IEEE, Akbar M. Sayeed, Member, IEEE, and Behnaam Aazhang, Fellow, IEEE
}

\begin{abstract}
The mobile wireless channel affords inherent diversity to combat the effects of fading. Existing code-division multiple-access systems, by virtue of spread-spectrum signaling and RAKE reception, exploit only part of the channel diversity via multipath combination. Moreover, their performance degrades under fast fading commonly encountered in mobile scenarios. In this paper, we develop new signaling and reception techniques that maximally exploit channel diversity via joint multipath-Doppler processing. Our approach is based on a canonical representation of the wireless channel, which leads to a time-frequency generalization of the RAKE receiver for diversity processing. Our signaling scheme facilitates joint multipath-Doppler diversity by spreading the symbol waveform beyond the intersymbol duration to make the channel time-selective. A variety of detection schemes are developed to account for the intersymbol interference (ISI) due to overlapping symbols. However, our results indicate that the effects of ISI are virtually negligible due to the excellent correlation properties of the pseudorandom codes. Performance analysis also shows that relatively small Doppler spreads can yield significant diversity gains. The inherently higher level of diversity achieved by time-selective signaling brings the fading channel closer to an additive white Gaussian noise channel, thereby facilitating the use of powerful existing coding techniques for Gaussian channels.
\end{abstract}

Index Terms-Diversity methods, Doppler, fading channels, multipath channels, spread-time signaling, time-frequency RAKE.

\section{INTRODUCTION}

$\mathbf{M}$ ULTIPATH fading caused by the wireless channel has a tremendous effect on the performance of mobile communication systems. Diversity techniques, which essentially amount to transmitting the signal over independent fading channels, are used in practice to combat fading [1]. Typical examples of diversity include time, frequency, polarization, and antenna (spatial) diversity.

The mobile wireless channel affords inherent diversity, which can be exploited via appropriate signaling and reception. Code-division multiple-access (CDMA) systems, by virtue of their spread-spectrum nature, have a remarkable ability to exploit channel diversity. The RAKE receiver at the heart

Paper approved by K. B. Letaief, the Editor for Wireless Systems of the IEEE Communications Society. Manuscript received July 18, 1998; revised December 13, 1998 and February 26, 1999. This work was supported by the National Science Foundation and the Texas Advanced Technology Program. This paper was presented in part at the IEEE International Symposium on Information Theory, Cambridge, MA, August 1998.

S. Bhashyam and B. Aazhang are with the Department of Electrical and Computer Engineering, Rice University, Houston, TX 77251-1892 USA.

A. M. Sayeed is with the Department of Electrical and Computer Engineering, University of Wisconsin-Madison, Madison, WI 53706 USA.

Publisher Item Identifier S 0090-6778(00)00493-1. of state-of-the-art CDMA systems utilizes the large signal bandwidth to exploit multipath diversity [1]-[3]. However, the RAKE structure is optimal for slow fading scenarios, and its performance degrades significantly in the presence of rapid temporal channel variations due to channel estimation errors [4], [5]. Such temporal channel variations are commonly encountered in mobile scenarios.

Temporal channel variations, in fact, provide another form of diversity-Doppler diversity-that can be exploited in conjunction with multipath to attain joint multipath-Doppler diversity [6]-[8]. The concept of joint multipath-Doppler diversity is based on a canonical channel representation [6]-[8] that decomposes the channel into a series of independent flat fading channels corresponding to different (orthogonal) multipath-Doppler-shifted signal components. Diversity reception is achieved by a generalization of the RAKE receiver that performs joint multipath-Doppler processing. The level of diversity is directly proportional to time-bandwidth product of the signaling waveform-multipath diversity is proportional to the bandwidth and Doppler diversity is proportional to the signaling duration. Thus, maximal exploitation of channel diversity dictates that spread-spectrum frequency-selective signaling should be complemented by maximally spreading the transmitted signal in time, as well to make the channel time-selective [6]-[8].

In this paper, we introduce a novel framework for time-selective signaling and reception techniques to achieve maximal channel diversity via joint multipath-Doppler processing. For simplicity of exposition, we restrict most of the discussion to the single-user case and binary phase-shift keying signaling in this paper. One simple extension to the multiuser scenario is illustrated in Section V-C. Extensions to $M$-ary and orthogonal signaling are also possible. The basic idea behind our signaling approach is to achieve time-spreading by increasing the symbol duration - symbols may overlap in time in order to keep the data rate constant. In effect, time-selective signaling facilitates maximal exploitation of Doppler diversity at the cost of introducing controlled intersymbol interference (ISI).

The front-end processing at the receiver is performed by a generalized time-frequency RAKE receiver, which correlates the received waveform with multipath-Doppler-shifted copies of the transmitted symbol waveform. Even though optimal reception requires sequence decoding, our performance results show that the effects of ISI due to overlapping symbols are virtually negligible. This lack of ISI can be attributed to the excellent correlation properties of pseudorandom codes, which improve 
arbitrarily with increasing length of the codes. Thus, for most practical scenarios, a one-shot detector that ignores ISI may be used at the receiver.

For the wide-sense stationary uncorrelated scatterer (WSSUS) channel, the level of diversity increases arbitrarily with the signaling duration. In fact, for coherent reception and under mild conditions on the channel, our approach asymptotically transforms the fading channel into an additive white Gaussian noise (AWGN) channel as the signaling duration increases. A similar result has been shown in [9] for the case of independently, identically distributed (i.i.d.) spatial diversity channels. The convergence result suggests that the performance of the proposed schemes could be further improved by using existing coding schemes developed for the Gaussian channel.

In practice, the maximum level of channel diversity achievable via time-selective signaling is limited by the maximum acceptable delay. In this context, a remarkable fact that endorses the practical viability of the proposed approach is that even the relatively small Doppler spreads encountered in practice can be leveraged into significant diversity gains with moderate time-spreading, which does not entail an unacceptable delay. Such diversity gains also facilitate effective use of simple coding schemes for the fading channel. Indeed, simulations results under realistic channel conditions illustrate that using a simple convolutional code in conjunction with our joint multipath-Doppler diversity approach can yield substantially improved performance compared to a coded system which only exploits multipath diversity as in conventional systems. Moreover, as suggested by the convergence result, the coding gain increases with increased diversity. Finally, simulation results also show that time-selective signaling provides a powerful alternative to interleaving. For a simple convolutional code, the coded time-selective signaling and reception scheme performs substantially better than a block-interleaved coded scheme under comparable delays.

We note that a system design approach similar to the one presented in this paper has been recently proposed in [10] and [11]. In particular, the signaling strategy proposed in this paper is very similar in spirit to the one in [10] and [11], except that our scheme employs standard binary spreading codes as opposed to the real-valued codes used in [10] and [11]. However, our receiver structure, inspired by the canonical multipath-Doppler channel decomposition, is fundamentally different from the design proposed in [10]. As we will see, the overall system proposed in this paper is optimally matched to the fast fading channel and can deliver improved performance compared to the scheme presented in [10]. Overlapping signature sequences have also been studied in [12] for other advantageous system characteristics, such as increased resistance to impulse noise and increased number of available signature sequences.

The remainder of the paper is organized as follows. The channel representation, which precisely reveals the channel diversity and lays the foundation of our approach, is presented in the next section. The signaling scheme for maximally exploiting channel diversity is described in Section III. Various reception techniques are developed in Section IV, and their performance is discussed in Section V. Section VI discusses the integration of proposed diversity schemes with coding.

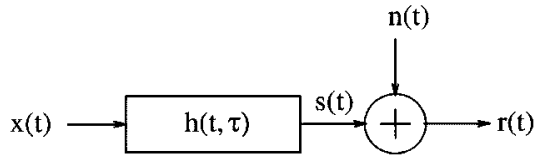

Fig. 1. Mobile wireless channel-linear time-varying system.

Conclusions and possible directions for future research are presented in the Section VII.

\section{CANONiCAl Channel RePresentation}

The determination of optimal modulation and demodulation techniques critically depends on accurate channel characterization. In this section, we present a canonical characterization of the mobile wireless CDMA channel developed in [6] and [7], which guides the development of our time-selective signaling scheme.

The received signal at the output of a mobile wireless channel is a superposition of multiple signals arriving from different paths that generally have different delays and attenuation. Additionally, the characteristics of the different paths vary with time due to the relative motion between transmitter and receiver. Thus, a mobile wireless channel can be generally described as a time-varying linear system. The signal at the output of the channel, depicted in Fig. 1, can be expressed as [11], [13]

$$
r(t)=s(t)+n(t)=\int_{0}^{T_{m}} h(t, \tau) x(t-\tau) d \tau+n(t)
$$

where $x(t)$ is the transmitted signal, $h(t, \tau)$ is the time-varying channel impulse response, and $n(t)$ is AWGN. The variable $\tau$ represents the multipath delay, and $T_{m}$ denotes the multipath spread of the channel.

An equivalent channel representation in terms of the spreading function $H(\theta, \tau)$ is

$$
s(t)=\int_{0}^{T_{m}} \int_{-B_{d}}^{B_{d}} H(\theta, \tau) x(t-\tau) e^{j 2 \pi \theta t} d \theta d \tau
$$

where $\theta$ corresponds to Doppler shifts introduced by the channel and $B_{d}$ denotes the Doppler spread of the channel. The spreading function is related to the impulse response via a Fourier transform and quantifies the time-frequency spreading produced by the channel [1], [13].

The time-varying impulse response $h(t, \tau)$ is best modeled as a stochastic process to account for channel variations. The WSSUS model [1], [13] in which $h(t, \tau)$ is represented as a stationary complex Gaussian process for each $\tau$ is widely used. Moreover, the channel responses at different delays $(\tau)$ are uncorrelated in the WSSUS model. Thus, the channel is characterized by second-order statistics given by ${ }^{1}$

$$
\frac{1}{2} E\left\{H\left(\theta_{1}, \tau_{1}\right) H^{*}\left(\theta_{2}, \tau_{2}\right)\right\}=\Psi\left(\theta_{1}, \tau_{1}\right) \delta\left(\theta_{1}-\theta_{2}\right) \delta\left(\tau_{1}-\tau_{2}\right)
$$

where

$$
\Psi(\theta, \tau) \triangleq \frac{1}{2} E\left\{|H(\theta, \tau)|^{2}\right\}
$$

\footnotetext{
${ }^{1}$ Assuming a zero-mean process, which corresponds to Rayleigh fading.
} 
is the scattering function of the channel. $T_{m}$ and $B_{d}$ correspond to the maximum supports of $\Psi(\theta, \tau)$ in the multipath and Doppler variables, respectively.

The WSSUS channel affords inherent diversity, which can be exploited with appropriate signaling and reception. The approach developed in this paper is inspired by the following canonical representation [6], [7] of the received signal corresponding to a single transmitted symbol

$$
\begin{aligned}
r(t) & \approx \frac{1}{T_{s} B} \sum_{n=0}^{N} \sum_{k=-K}^{K} \hat{H}\left(\frac{k}{T_{s}}, \frac{n}{B}\right) u_{n, k}(t)+n(t) \\
u_{n, k}(t) & =q\left(t-\frac{n}{B}\right) e^{j\left(2 \pi k t / T_{s}\right)}, \quad N=\left\lceil T_{m} B\right\rceil \\
K & =\left\lceil B_{d} T_{s}\right\rceil
\end{aligned}
$$

where $q(t)$ is the transmitted spread-spectrum signaling waveform of duration $T_{s}$ and bandwidth $B$. $\lceil x\rceil$ denotes the smallest integer greater than or equal to $x . \hat{H}(\theta, \tau)$ denotes a time-frequency smoothed version of $H(\theta, \tau)$ which comes about due to the finite duration and effectively finite bandwidth of the transmitted symbol [7].

For direct-sequence CDMA systems, the bandwidth $B \propto 1 / T_{c}$, where $T_{c}$ is the chip duration. In this paper, we consider a chip-rate sampled system corresponding to the approximation $B=1 / T_{c}$, resulting in the channel coefficients $\hat{H}\left(\left(k / T_{s}\right), n T_{c}\right)$ and

$$
u_{n, k}(t)=q\left(t-n T_{c}\right) e^{j\left(2 \pi k \cdot t / T_{s}\right)}
$$

in (5). We note that the main source of error in (5) is due to the bandwidth approximation, which can be made arbitrarily small by oversampling (sub-chip-rate) in time [7], [14].

The representation (5) clearly reveals the inherent channel diversity. The multipath-Doppler-shifted waveforms $\left\{u_{n, k}(t)\right\}$ are (approximately) orthogonal, and the channel samples $\hat{H}\left(k / T_{s}, n T_{c}\right)$ are (approximately) independent random variables [7]. Each waveform $u_{n, k}(t)$ serves as a diversity channel thereby facilitating $(N+1)(2 K+1)$-level joint multipath-Doppler diversity. Since the diversity level is proportional to the time-bandwidth product (TBP) of the signaling waveform, spread-spectrum CDMA systems are particularly suited for exploiting the inherent channel diversity.

\section{Time-Selective SignaLing}

The canonical channel representation (5) suggests that for maximal exploitation of channel diversity, the symbol duration $T_{s}$ should be increased as much as possible. This is the basic idea behind our approach to time-selective signaling. Spreading waveforms of duration $T_{s}$ are employed that typically last longer than the intersymbol duration $T$ determined by the data rate, thereby introducing overlap between symbols. The transmitted signal $x(t)$ can be represented as

$$
x(t)=\sqrt{E_{s}} \sum_{i} b^{i} q(t-i T)
$$

where $b^{i}( \pm 1)$ are the transmitted symbols, $E_{s}$ is the signal energy per symbol, $1 / T$ is the data rate, and $T_{s} \geq T$ is the

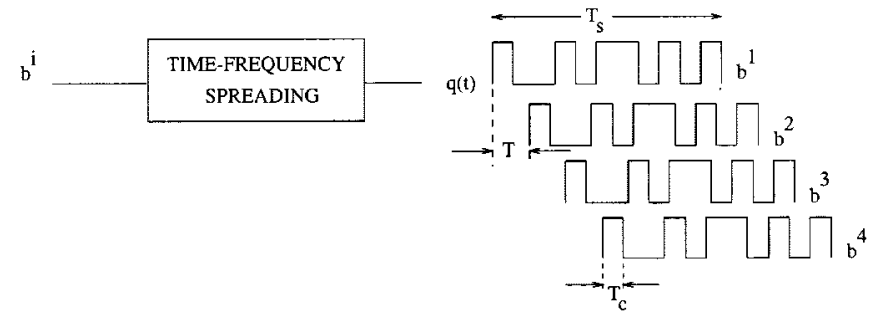

Fig. 2. Time-selective signaling.

time-support of the spreading waveform $q(t)$. This time-selective signaling scheme is illustrated in Fig. 2. As shown in Fig. 2, each of the symbols is spread in frequency to a bandwidth $B \approx$ $1 / T_{c}$ to exploit multipath diversity by making the channel frequency-selective, and in time to a duration $T_{s}$ to exploit Doppler diversity by making the channel time-selective.

Using (5), which represents the received signal for a single symbol, the received signal corresponding to the transmitted signal $x(t)$ in (7) can be expressed as

$$
\begin{aligned}
& r(t) \approx \sqrt{E_{s}} \frac{T_{c}}{T_{s}} \sum_{i} b^{i} \sum_{n=0}^{N} \sum_{k=-K}^{K} \hat{H}^{i} \\
& \cdot\left(\frac{k}{T_{s}}, n T_{c}\right) u_{n, k}^{i}(t)+n(t)
\end{aligned}
$$

where $\hat{H}^{i}\left(k / T_{s}, n T_{c}\right)$ denote the channel coefficients and $u_{n, k}^{i}(t)=u_{n, k}(t-i T)$ the multipath-Doppler-shifted signaling waveforms for the $i$ th symbol. Note that the number of Doppler components, $2 K+1$, is proportional to the signaling duration $T_{s}$. Thus, time-selective signaling facilitates maximal exploitation of channel diversity at the expense of introducing controlled ISI. In practice, the amount of achievable diversity is limited by delay constraints since a delay of $T_{S}$ is introduced at the receiver.

\section{RECEPTION TECHNIQUES}

In this section, we discuss reception techniques for the signaling scheme proposed in Section III. For all our detectors, we will assume perfect knowledge of the channel coefficients. ${ }^{2}$ Due to ISI, optimal detection requires that all symbols be detected jointly, since at the very least, each symbol overlaps with the preceding and succeeding symbols. However, as we will see, the effects of ISI are relatively negligible due to the excellent autocorrelation properties of pseudorandom codes. Thus, a "oneshot" detector, which ignores ISI and employs maximal-ratio diversity combining, may be used at the receiver for most practical situations.

\section{A. Optimal Detector}

We first derive the optimal maximum-likelihood sequence detector [18]-[20]. Conditioned on the channel coefficients and

\footnotetext{
${ }^{2} \mathrm{~A}$ pilot signal may be used to estimate these channel coefficients. This can be done in principle as long as the channel is underspread, i.e., $B_{d} T_{m}<(1 / 2)$ [15], [16]. Most practical channels are underspread [1], [17], including the examples discussed in this paper. A thorough analysis of the effect of estimation errors on performance is beyond the scope of this paper.
} 
the symbol sequence, the log-likelihood function can be written as

$$
\begin{aligned}
& \Lambda\left(r(t) /\left\{h_{n, k}^{i}\right\},\left\{b^{i}\right\}\right) \\
& =-\int_{-\infty}^{\infty}\left|r(t)-\sum_{i} \sum_{n, k} b^{i} h_{n, k}^{i} u_{n, k}^{i}(t)\right|^{2} d t
\end{aligned}
$$

where $h_{n, k}^{i} \triangleq\left(T_{c} / T_{s}\right) \hat{H}^{i}\left(\left(k / T_{s}\right), n T_{c}\right)$. Neglecting the terms independent of the symbol sequence, we can reduce this expression to obtain an iterative metric given by [18], [20]

$$
\begin{aligned}
\sum_{i=-\infty}^{\infty} 2 \operatorname{Re} & \left\{\sum_{n, k}\left(b^{i} z_{n, k}^{i} h_{n, k}^{i^{\star}}\right)\right. \\
& \left.-\sum_{l=i-I+1}^{i-1} b^{i} b^{l} \sum_{n^{\prime}, k^{\prime}, n, k} h_{n^{\prime}, k^{\prime}}^{i^{\star}} h_{n, k}^{l} R_{n^{\prime}, k^{\prime}, n, k}^{i, l}\right\}
\end{aligned}
$$

where

$$
\begin{aligned}
z_{n, k}^{i}= & \int_{i T+n T_{c}}^{i T+n T_{c}+T_{s}} r(t) u_{n, k}^{i^{*}}(t) d t \\
= & \int_{i T+n T_{c}}^{i T+n T_{c}+T_{s}} r(t) q^{*}\left(t-i T-n T_{c}\right) \\
& \quad \cdot \exp \left(-j \frac{2 \pi k(t-i T)}{T_{s}}\right) d t
\end{aligned}
$$

are the correlator outputs corresponding to the multipath-Doppler-shifted versions of the $i$ th transmitted symbol, and

$$
\begin{aligned}
R_{n^{\prime}, k^{\prime}, n, k}^{i, l}= & \int_{-\infty}^{\infty} u_{n^{\prime}, k^{\prime}}^{i^{\star}}(t) u_{n, k}^{l}(t) d t \\
= & \int_{-\infty}^{\infty} q\left(t-l T-n T_{c}\right) \\
& \cdot \exp \left(j \frac{2 \pi k(t-l T)}{T_{s}}\right) q^{\star}\left(t-i T-n^{\prime} T_{c}\right) \\
& \cdot \exp \left(-j \frac{2 \pi k^{\prime}(t-i T)}{T_{s}}\right) d t
\end{aligned}
$$

is the correlation between the multipath-Doppler waveforms for the $i$ th and $l$ th symbols. It is evident from the optimal metric in (10) that given the channel coefficients, the matched filter outputs $z_{n, k}^{i}$ corresponding to the multipath-Doppler signal components constitute the sufficient statistics for reception. In fact, the matched filter outputs $z_{n, k}^{i}$, defined in (11), perform a timefrequency correlation and are a generalization of the tappeddelay line RAKE structure [1] to fully account for fast fading channel characteristics [6], [7]. Furthermore, the matched filter outputs $z_{n, k}^{i}$ can be efficiently computed with a bank of conventional RAKE receivers each matched to a different Doppler frequency $(k)$ [7].

The representation of the metric (10) as a summation over $i$, where each term of the summation can be computed at time interval $i$, permits an implementation using the Viterbi algorithm. The value of $I$ is determined by the number of symbols that overlap due to spreading in time. A trellis can be built with $2^{I-1}$ states and a trellis-based sequence detection can be performed. Although the sequence detection technique is optimal, it suffers from prohibitive computational complexity since the number of states is exponential in the number of symbols that overlap, and for maximum diversity gain, we need to use as large an overlap as possible. Thus, we next explore suboptimal linear detectors that are computationally tractable and more desirable in practice.

\section{B. Suboptimal Linear Detectors}

We consider a detection window of $2 I+1$ symbols $(-I \leq i \leq$ $I)$ centered around the symbol of interest, which we consider to be the zeroth symbol without loss of generality. To develop suboptimal receivers, we rewrite the matched-filter outputs of the time-frequency RAKE receiver (11) conveniently in matrix notation as

$$
\boldsymbol{z}=\boldsymbol{R} \boldsymbol{H b}+\boldsymbol{n}
$$

where $z$ is the $(2 I+1)(N+1)(2 K+1) \times 1$ vector of sufficient statistics containing the outputs of the time-frequency RAKE receiver for the different symbols, $\boldsymbol{b}$ is the $(2 I+1) \times 1$ vector of symbols, $\boldsymbol{n}$ is the $(2 I+1)(N+1)(2 K+1) \times 1$ vector of noise components at the outputs of each of the matched filters, and $\boldsymbol{R}$ is a $(2 I+1)(N+1)(2 K+1) \times(2 I+1)(N+1)(2 K+1)$ correlation matrix defined as

$$
\boldsymbol{R}=\left[\begin{array}{cccc}
\boldsymbol{R}_{-I,-I} & \boldsymbol{R}_{-I,-I+1} & \cdots & \boldsymbol{R}_{-I, I} \\
\boldsymbol{R}_{-I+1,-I} & \boldsymbol{R}_{-I+1,-I+1} & \cdots & \boldsymbol{R}_{-I+1, I} \\
\vdots & \vdots & \vdots & \vdots \\
\boldsymbol{R}_{I,-I} & \boldsymbol{R}_{I,-I+1} & \cdots & \boldsymbol{R}_{I, I}
\end{array}\right]
$$

where

$$
\boldsymbol{R}_{i, j}=\int_{-I T}^{I T+T_{s}} u^{i *}(t) \boldsymbol{u}^{j T}(t) d t .
$$

The channel coefficients are represented by the $(2 I+1)(N+$ 1) $(2 K+1) \times(2 I+1)$ matrix

$$
\boldsymbol{H}=\left[\begin{array}{cccc}
h^{-I} & 0 & \cdots & 0 \\
0 & h^{-I+1} & 0 & \cdots \\
\vdots & \vdots & \ddots & \vdots \\
0 & \cdots & 0 & \boldsymbol{h}^{I}
\end{array}\right]
$$

where $h^{i}$ is the $(N+1)(2 K+1) \times 1$ vector of channel coefficients for the $i$ th symbol.

The expression for time-frequency RAKE outputs in (13) is similar in form to the front-end matched-filter outputs for ISI or multiaccess interference channels, and thus, techniques similar to those developed in [21]-[24], [1], [19] can be applied here. We restrict our discussion to a linear technique that is an analog of the decorrelating detector [23], [21]

$$
\hat{\boldsymbol{b}}=\operatorname{sign}\left\{\operatorname{Re}\left(\boldsymbol{H}^{H} \boldsymbol{D} \boldsymbol{L} z\right)\right\}
$$

where $\boldsymbol{L}=\boldsymbol{R}^{-1}$ removes the ISI and $\boldsymbol{D}$ performs a whitening operation. Due to ISI removal by $L$, the noise at the output of the zero-forcing stage is enhanced and is correlated across the various diversity branches for each symbol. Therefore, the 
noise-whitening stage $\boldsymbol{D}$ is applied to the output before maximal-ratio combining and is a block diagonal matrix given by [21], [22], [25]

$$
\boldsymbol{D}=\left[\begin{array}{cccc}
\left(\boldsymbol{Q}_{-I}\right)^{-1} & 0 & \cdots & 0 \\
0 & \left(\boldsymbol{Q}_{-I+1}\right)^{-1} & 0 & \cdots \\
\vdots & \vdots & \ddots & \vdots \\
0 & \cdots & 0 & \left(\boldsymbol{Q}_{I}\right)^{-1}
\end{array}\right]
$$

where $\boldsymbol{Q}=\boldsymbol{R}^{-1}$ and $\boldsymbol{Q}_{i}$ corresponds to the block of $\boldsymbol{Q}$ corresponding to the $i$ th symbol. $\boldsymbol{H}^{H}$ performs maximal-ratio combining. The decision for the middle symbol of the block is obtained from $\hat{\boldsymbol{b}}$ after which the window is moved by one symbol to detect the next symbol.

We will see from the performance analysis and the simulation results that the effects of ISI are virtually negligible compared to the diversity gains in time-selective signaling. This motivates the use of a simple one-shot detector which ignores ISI and performs simple maximal-ratio combining

$$
\hat{\boldsymbol{b}}=\operatorname{sign}\left\{\operatorname{Re}\left(\boldsymbol{H}^{H} \boldsymbol{z}\right)\right\} \text {. }
$$

The one-shot detector in (19) can be efficiently computed with a bank of conventional RAKE receivers [7].

The reception techniques developed in this section, in combination with the signaling scheme proposed in Section III, constitute the proposed system for maximally exploiting channel diversity via multipath-Doppler processing.

\section{PeRformance AnAlysis}

In this section, we assess the performance of the proposed system under realistic fast fading scenarios. Our discussion shows that time-selective signaling can yield significant gains for practical Doppler spreads and asymptotically transforms the fading channel into a marginally AWGN channel. Moreover, for most practical scenarios, ISI is virtually negligible due to the excellent correlation properties of pseudorandom codes, and thus, the one-shot time-frequency RAKE structure (19) can be employed at the receiver.

\section{A. Diversity Gains}

Recall from the canonical representation (5) that the number of Doppler diversity components is proportional to the product $B_{d} T_{s}$. We begin by illustrating that the relatively small Doppler spreads $B_{d}$ encountered in practice can be leveraged into significant diversity gains via moderate time-spreading which yields $B_{d} T_{s} \approx 0.1-0.2$. It is worth noting that at such values of $B_{d} T_{s}$, the signal energy captured by the $k= \pm 1$ Doppler matched filters, corresponding to the projection of the signal onto the Doppler-shifted waveforms, is about $1 \%-2 \%$ of the total signal energy. Such Doppler diversity gains at relatively small values of $B_{d} T_{s}$ are due to the nonlinear dependence of diversity gain on the energy distribution in the various diversity components - a significant fraction of the maximum diversity gain due to an additional diversity component is attained at a very small fraction of the total energy in that component [7]. Fig. 3 illustrates this phenomenon by depicting the performance

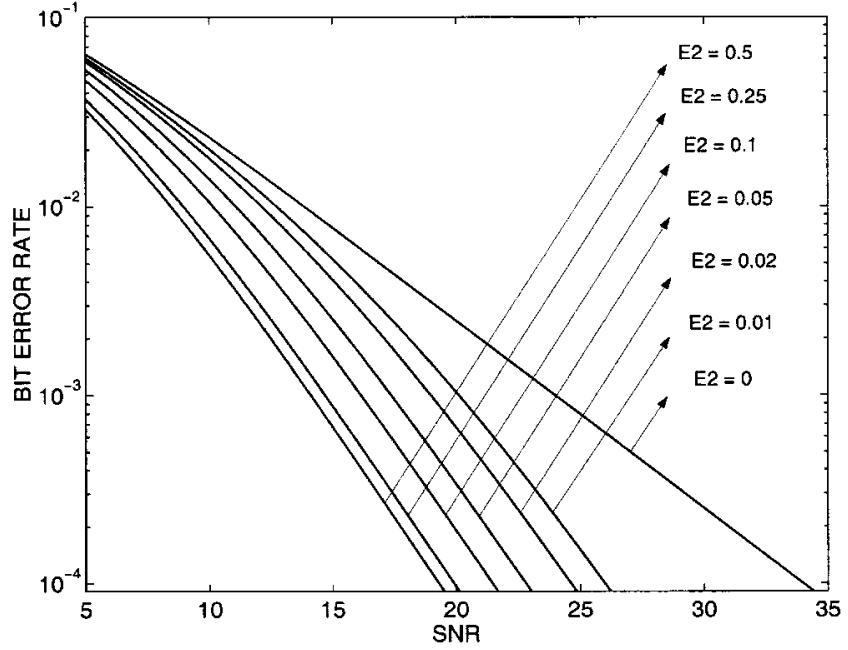

Fig. 3. Bit-error rate (BER) versus signal-to-noise ratio (SNR) per symbol for a two-branch diversity system- $-E_{1}$ and $E_{2}$ are the average energies in branches 1 and 2, respectively, and $E_{1}+E_{2}=1$.

of a system with two diversity branches under varying proportions of energies in the branches. The bit-error probability $\left(P_{e}\right)$ is calculated analytically [1] based on an idealized system with no ISI. It is evident that most of the performance gain is achieved with $1-2 \%$ of the energy in the additional diversity component $\left(E_{2}=0.01 \%-0.02\right)$, and further increase in the relative energy of the second component exhibits diminishing returns in the diversity gain. We note that in practice, reliable estimation of channel coefficients corresponding to weaker diversity components is more difficult compared to those for stronger diversity components, and such issues must be taken into account in the final design.

\section{B. Performance of the Decorrelating Detector}

In this section, we derive an expression for the bit-error probability of the decorrelating receiver, which will be later used to assess the simulation results and the effects of ISI. As seen in Section IV-B, the decorrelating detector has three stages-decorrelation, noise-whitening, and diversity combining. At the output of the decorrelating stage, there is no interference between the different overlapping symbols, and the noise is distributed as Gaussian $\left(\mathbf{o}, R^{-1}\right)$. The output block (of multipath-Doppler components) corresponding to a symbol of interest (say $i$ th symbol) is corrupted by a noise vector that is Gaussian $\left(\mathbf{o},\left(\boldsymbol{R}^{-1}\right)_{i}\right)$, where $\left(\boldsymbol{R}^{-1}\right)_{i}$ is the block of $R^{-1}$ corresponding to the $i$ th symbol. The noise-whitening operation is performed as given by (17) and (18). The bit-error probability after combining these diversity branches is given by [21], [22], [25]

$$
P_{e}=\frac{1}{2} \sum_{l=1}^{(N+1)(2 K+1)} \pi_{l}\left[1-\sqrt{\frac{\gamma_{l}}{1+\gamma_{l}}}\right]
$$

where $\pi_{l}$ is defined as

$$
\pi_{l}=\prod_{i=1, i \neq l}^{(N+1)(2 K+1)} \frac{\lambda_{l}}{\lambda_{l}-\lambda_{i}}
$$




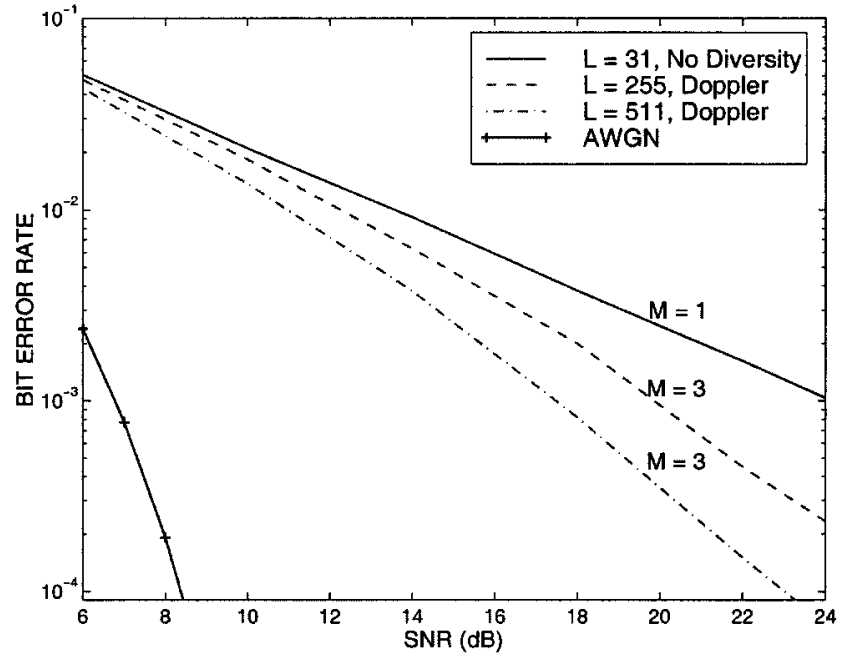

Fig. 4. BER versus SNR per symbol—single path, data rate $=10 \mathrm{kHz}$, length of spreading code $=L$, intersymbol duration $=T / T_{c}=31$, mobile speed $=$ $50 \mathrm{mi} / \mathrm{h} . M$ denotes the total number of diversity channels.

where $\gamma_{l}=\lambda_{l} E_{s} / \mathcal{N}_{0}$ is the effective average SNR for the $l$ th diversity branch, and $\lambda_{l}$ is the $l$ th distinct eigenvalue of $\boldsymbol{\Phi}_{i}\left(\boldsymbol{R}^{-1}\right)_{i}^{-1}$ where $\boldsymbol{\Phi}_{\boldsymbol{i}}=E\left[\boldsymbol{h}^{i} \boldsymbol{h}^{i^{H}}\right]$ is the covariance matrix of the multipath-Doppler channel coefficients corresponding to the $i$ th symbol.

\section{Simulation Results}

We now present some simulation results on the performance of the techniques for time-selective signaling and reception proposed in Sections III and IV and discuss their implications. A spread-spectrum system with the RAKE receiver with length $L=\left(T_{s} / T_{c}\right)=31$ (or 15) spreading is used as the baseline for comparison. Time-selective signaling systems employing timefrequency RAKE receivers with overlapping codes of length $L=255$ and 511, with a new symbol transmitted every 31 (or 15) chips, are compared with the baseline system. The timeselective systems achieve $B_{d} T_{s}$ of approximately 0.1 and 0.2 for code lengths 255 and 511, respectively. The Jakes fading channel model with a carrier frequency of $1.8 \mathrm{GHz}$ and mobile speed of 50 (or 100) $\mathrm{mi} / \mathrm{h}$ is used to simulate the channel at a data rate of $10 \mathrm{kHz}$.

Figs. 4 and 5 summarize the simulated performance of the decorrelating receiver for the two systems for different number of multipath components. The total number of diversity components $(M=(N+1)(2 K+1))$ in each case is also indicated in the figures. Fig. 4 corresponds to a single-path fading channel $(N+1=1)$, whereas Fig. 5(a) and (b) shows results for a frequency-selective fading channel with two paths $(N+1=2)$ corresponding to mobile speeds of 50 and $100 \mathrm{mi} / \mathrm{h}$, respectively. The number of Doppler diversity components $(2 K+1)$ is 1,3 , and 3 for $L=31$ (or 15), 255, and 511 respectively. Although, the number of diversity components is the same for $L=255$ and 511, they differ in performance because they have different energy distributions across the diversity components. The performance of a fading channel with no diversity and an AWGN channel are also shown in all the three figures for comparison. As evident from the figures, time-selective signaling and reception can deliver significant gains by exploiting joint multi-

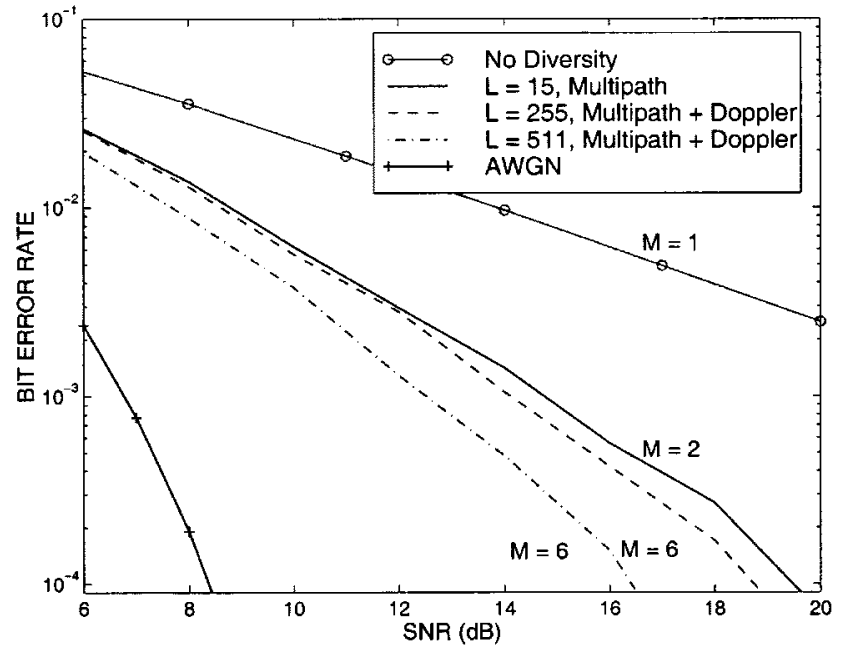

(a)

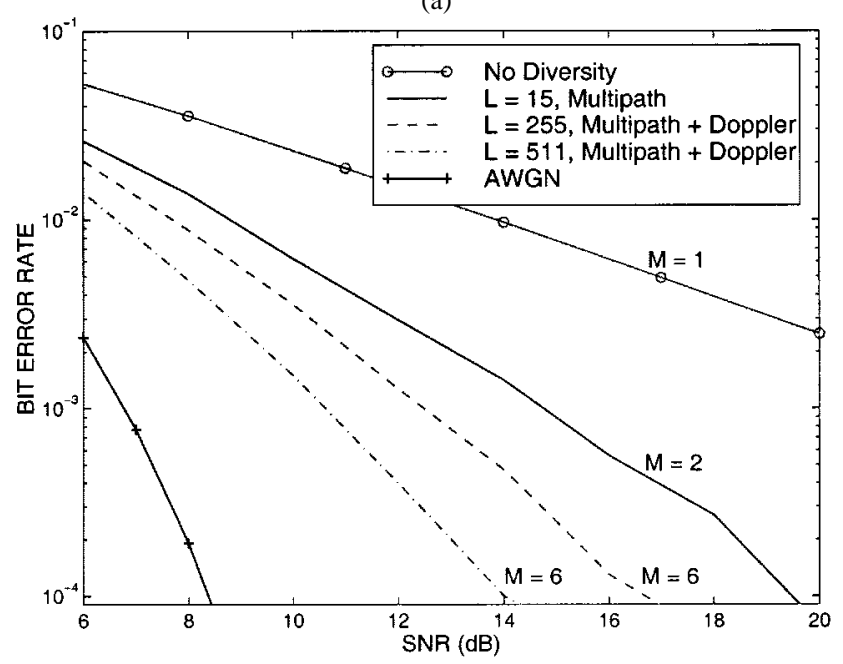

(b)

Fig. 5. BER versus SNR per symbol-two paths, data rate $=10 \mathrm{kHz}$, length of spreading code $=L$, intersymbol duration $=T / t_{c}=15$, ratio of average energy of the two paths $=2: 1$, (a) mobile speed $=50 \mathrm{mi} / \mathrm{h}$ and (b) mobile speed $=100 \mathrm{mi} / \mathrm{h} . M$ denotes the total number of diversity channels.

path-Doppler diversity. For example, in Fig. 4, the single-path system with $L=511(M=3)$ shows a 6-10-dB SNR improvement over the conventional system $(M=1)$ for $P_{e}$ between $10^{-3}$ and $10^{-4}$. It should be also noted that the performance of the $L=255$ system at $100 \mathrm{mi} / \mathrm{h} \mathrm{[Fig.} \mathrm{5(b)]} \mathrm{is} \mathrm{almost} \mathrm{the} \mathrm{same} \mathrm{as}$ the performance of the $L=511$ system at $50 \mathrm{mi} / \mathrm{h}$ [Fig. 5(a)]. This indicates that the performance gains attainable at $100 \mathrm{mi} / \mathrm{h}$ can be achieved at $50 \mathrm{mi} / \mathrm{h}$ by increasing the symbol duration $(L)$ by a factor of 2 to make the channel more time-selective.

Fig. 6 illustrates the relative insignificance of ISI in the proposed system by comparing the performance of the one-shot detector, the decorrelating detector, and an idealized system that does not suffer from ISI and achieves a comparable level of diversity. The theoretical performance of the decorrelating detector computed in Section V-B is also included for comparison. The channel statistics required to calculate $P_{e}$ for the decorrelating detector and the idealized detector were obtained from the simulated channel data. We note that the theoretical performance of the decorrelator follows the same trend as the performance of the idealized system with no ISI. Moreover, the 


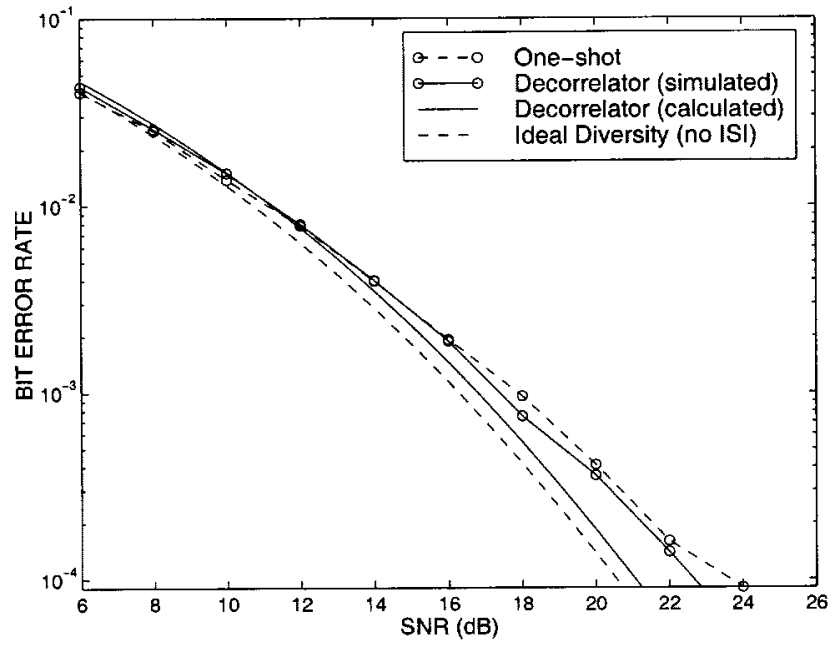

Fig. 6. Comparison of decorrelating detector with one-shot detector-BER versus SNR per symbol—data rate $=10 \mathrm{kHz}$, length of spreading code $=L=$ 511 , intersymbol duration $=T / T_{c}=31$, single path, mobile speed $=50 \mathrm{mi} / \mathrm{h}$ The total number of diversity channels is $M=3$.

performance of different systems is virtually identical at lower SNR's (relatively higher $P_{e}$ 's), and the theoretical performance curves begin to diverge from simulated curves with increasing SNR (lower $P_{e}$ 's), as the effects of interference become more pronounced compared to the AWGN.

The ISI introduced by overlapping codes diminishes with increasing length of the code. As the code length increases, the number of symbols which overlap increases, but the correlation properties of the code also improve, thereby resulting in reduced ISI contribution due to each overlapping symbol. Similar effects have also been reported in [26] in the context of multiuser interference. The excellent autocorrelation properties of spreading codes are illustrated in Fig. 7 which plots the magnitude of the time-frequency correlation function

$$
Q(n, k)=\int q(t) q\left(t-n T_{c}\right) e^{-\left(j 2 \pi k t / T_{s}\right)} d t
$$

for two different code lengths. The correlation values are much smaller for $L=511$ than for $L=31$. Thus, under realistic fading conditions and symbol-overlaps considered here, ISI is relatively insignificant in the proposed system. This suggests that the one-shot detector (19), which ignores ISI, suffices for reception.

Performance gains, implicitly due to Doppler diversity, have also been reported in other non-CDMA systems [20] for $B_{d} T_{s} \approx 0.1$. However, we note that nonspread spectrum systems have a limited ability to exploit channel diversity due to the small TBP $\approx 1$-they cannot exploit both multipath and Doppler diversity simultaneously. CDMA systems, on the other hand, have a unique ability to exploit joint multipath-Doppler diversity by virtue of attaining an arbitrarily large TBP via time-selective signaling by increasing the duration of the signaling waveform (while keeping the bandwidth fixed).

Perfect channel estimation has been assumed in all the cases shown. In practice, reliable channel estimation can be done in principle as long as the channel is underspread, i.e., $B_{d} T_{m}<$ 0.5 [15], [16]. In our case, the multipath spread, $T_{m}$ is, at most,

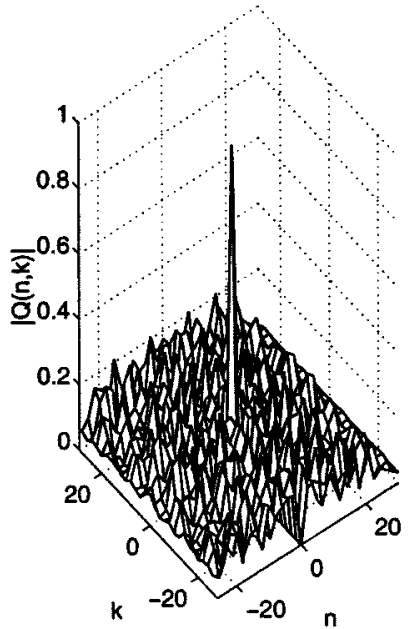

(a)

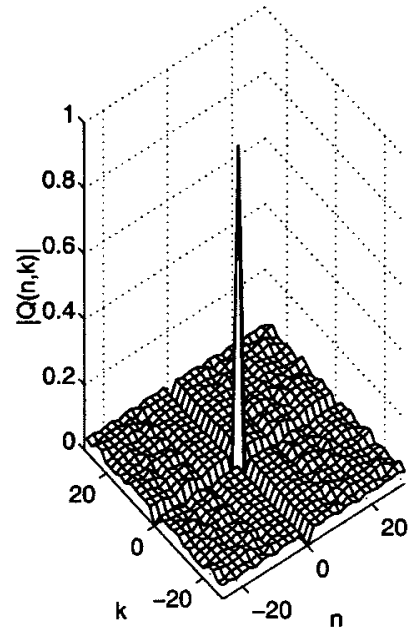

(b)
Fig. 7. Time-frequency correlation function of a typical spreading code ( $m$-sequence) for two different code lengths: (a) $L=31$ and (b) $L=511$.

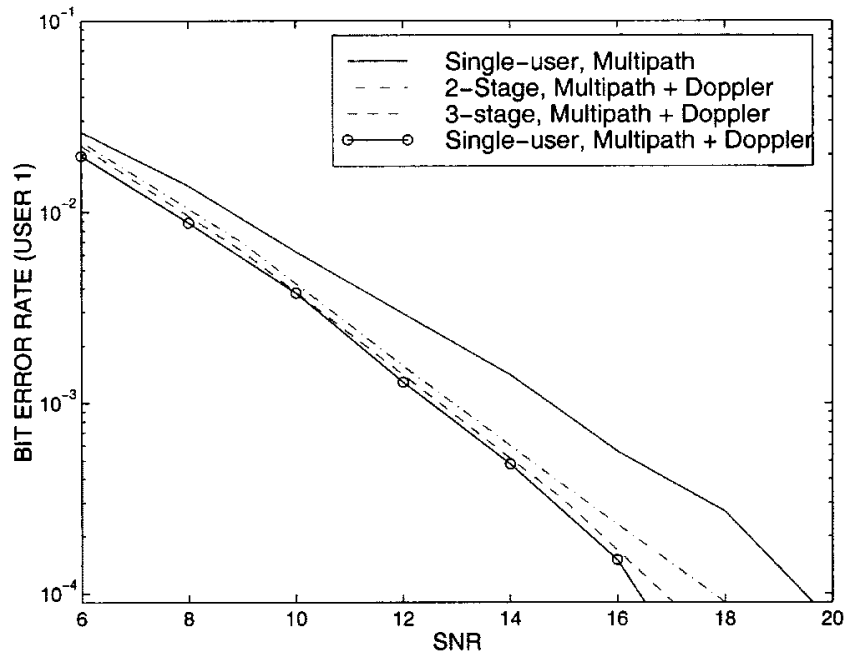

Fig. 8. BER versus SNR per symbol for the desired user in a four-user system-two paths, user of interest has $6 \mathrm{~dB}$ lower power than other users, data rate $=10 \mathrm{kHz}$, length of spreading code $=511$, intersymbol duration $=T / T_{c}=15$, mobile speed $=50 \mathrm{mi} / \mathrm{h}$. Single-user system performance has also been shown for reference. As evident, Doppler diversity gains are attained in the multiuser system as well.

$T_{c}(T / 15=0.0001 / 15)$ and the Doppler spread $B_{d}$ is approximately $133 \mathrm{~Hz}$ for a mobile speed of $50 \mathrm{mi} / \mathrm{h}$ and carrier frequency of $1.8 \mathrm{GHz}$. This translates into a channel spreading factor $B_{d} T_{m}$ of 0.00089 .

Finally, we illustrate using a simple example that these performance gains can be achieved in multiuser systems by appropriately combining the proposed diversity transceivers with multiuser detection techniques. Fig. 8 shows the performance of a four-user system with the interfering users being $6 \mathrm{~dB}$ stronger than the user of interest. Multistage detection [24] is used to combat multiple-access interference. The performance of the single-user receiver (which does not suffer from multiple-access interference), at the same SNR as that of the desired user, has also been shown for reference. It can be seen that the performance in the multiuser environment with near-far effect is almost the same as the performance in the single-user case. 


\section{Asymptotic Behavior of the System}

This section discusses the asymptotic behavior of the proposed system as the number of Doppler diversity components increases with signaling waveforms of longer duration.

Recall that the received signal is

$$
r(t)=\sqrt{E_{s}} \sum_{i} b^{i} \sum_{n=0}^{N} \sum_{k=-K}^{K} h_{n, k}^{i} u_{n, k}^{i}(t)+n(t)
$$

and the coherently combined outputs of the matched filters can be written as

$$
\begin{aligned}
Z^{i}=b^{i} S^{i}+N^{i}=b^{i} \sqrt{E_{s}} \sum_{n=0}^{N} & \sum_{k=-K}^{K}\left|h_{n, k}^{i}\right|^{2} \\
& +\sum_{n=0}^{N} \sum_{k=-K}^{K} h_{n, k}^{i^{\star}} n_{n, k}^{i}
\end{aligned}
$$

under the orthogonality assumption

$$
\left\langle u_{n, k}^{i}, u_{n^{\prime}}^{j}, k^{\prime}\right\rangle= \begin{cases}1, & \text { when }(i, n, k)=\left(j, n^{\prime}, k^{\prime}\right) \\ 0, & \text { else. }\end{cases}
$$

which is closely satisfied as seen earlier. For the asymptotic analysis, we consider the case where $T_{s} \rightarrow \infty(K \rightarrow \infty)$ with

$$
\sum_{n=0}^{N} \sum_{k=-K}^{K} E\left[\left|h_{n, k}^{i}\right|^{2}\right]=C \quad \text { (constant) }
$$

which ensures the realistic constraint that the average power in the channel is constant. In (24), $S^{i}$ has a mean $C \sqrt{E_{s}}$ and $N^{i}$ is zero mean with variance $C \mathcal{N}_{0}$. Thus, the average SNR is $C E_{s} / \mathcal{N}_{0}$, which would be the actual SNR of the corresponding AWGN channel in the absence of fading. Under mild constraints, as the number of Doppler diversity components increases, the fading channel converges to a marginally AWGN channel with the same SNR - for each symbol $i$, the signal term $S^{i}$ converges to its mean and the noise term $N^{i}$ converges to a zero-mean Gaussian random variable with variance $C \mathcal{N}_{0}$.

Proposition 1: Suppose that the zero-mean independent complex Gaussian random variables $\left\{h_{n, k}^{i}\right\}$ and $\left\{n_{n, k}^{i}\right\}$ satisfy the following two conditions.

1) The Lindeberg condition the for central limit theorem (CLT) [27] holds for the set of random variables $\left\{h_{n, k}^{i^{\star}}, n_{n, k}^{i}\right\}$.

2) The sum of the squares of the second moments of $\left\{h_{n, k}^{i}\right\}$ converges to zero

$$
\sum_{n=0}^{N} \sum_{k=-K}^{K} E^{2}\left[\left|h_{n, k}^{i}\right|^{2}\right] \rightarrow 0 .
$$

Then

$$
Z^{i} \rightarrow \operatorname{Gaussian}\left(C \sqrt{E_{s}}, C \mathcal{N}_{0}\right) \text { as } K \rightarrow \infty .
$$

Proof: See the Appendix.
The two conditions ensure convergence of the fading channel to a marginally AWGN channel with SNR $C E_{s} / \mathcal{N}_{0}$. The Lindeberg condition is a sufficient condition for CLT and it ensures the convergence of the noise term to a Gaussian random variable. It also implies that [27]

$$
\max _{k} \frac{E\left[\left|h_{n, k}^{i}\right|^{2}\right]}{\sum_{n=0}^{N} \sum_{k=-K}^{K} E\left[\left|h_{n, k}^{i}\right|^{2}\right]} \rightarrow 0,
$$

which means that asymptotically no diversity component has a finite fraction of the total received signal energy. The second condition ensures that the signal term converges to its mean. As a special case, it can be easily seen that i.i.d. $\left\{h_{n, k}^{i}\right\}$ satisfy all the conditions. This special case has already been shown in [9].

A similar convergence result has also been shown in [10], where the asymptotic behavior of a system with time-spread symbols is considered. In [10], the fading channel with infinite time-spreading (and hence, infinite temporal diversity) converges to a marginally Gaussian channel with a lower SNR than $C E_{s} / \mathcal{N}_{0}$ due to an additional noise variance term. However, the diversity signaling and reception based on the canonical channel representation enable our system to achieve the SNR $C E_{s} / \mathcal{N}_{0}$ of the corresponding nonfading AWGN channel. This difference in the asymptotic performance of our system compared to the one proposed in [10] is due to the fundamentally different reception approach adopted here. Our receiver structure, inspired by the canonical channel representation, is optimally matched to the fast fading channel (and may also be incorporated in the system in [10]).

\section{DIVERSITY AND CODING}

We saw in the last section that exploiting channel diversity via time-selective signaling asymptotically transforms the fading channel into an AWGN channel. This suggests the use of standard coding techniques developed for the AWGN channel in conjunction with time-selective signaling over fading channels. In this section, we illustrate the effect of multipath-Doppler diversity reception on coding via the example of a simple convolutional code. Using the same coding scheme, the proposed signaling and reception technique is also compared with a block interleaving scheme with a comparable delay.

\section{A. Effect of Diversity on Coding Gain}

A rate $r=1 / 2$, constraint length $l=3$ convolutional code is chosen to illustrate the relationship between diversity and coding. The choice of the code is based on its computational simplicity. The performance of two coded systems is compared: a length $L=511$ proposed system employing the time-frequency RAKE receiver and a baseline RAKE receiver corresponding to a length $L=15$ spreading code. Soft-decision Viterbi decoding is used, where the soft-decision is obtained by coherently combining the RAKE receiver outputs. The number of diversity components in the various examples are the same as in Section V-C. Per the discussion in Section V-C, ISI effects are ignored. 
TABLE I

CODING AND DIVERSITY GAINS AT $P_{e}=10^{-3}$

\begin{tabular}{|c|c|c|c|c|c|}
\hline \multicolumn{6}{|c|}{ Coding Gains (dB) at $P_{e}=10^{-3}$} \\
\hline \multirow{2}{*}{$\begin{array}{c}\text { Data Rate } \\
(\mathrm{kbps})\end{array}$} & \multirow{2}{*}{$\begin{array}{c}\text { Mobile Speed } \\
(\mathrm{miles} / \mathrm{hr})\end{array}$} & \multicolumn{2}{|c|}{1 Path } & \multicolumn{2}{|c|}{2 Paths } \\
\hline & & $L=15$ & $L=511$ & $L=15$ & $L=511$ \\
\hline 10 & 50 & 5.30 & 1.76 & 2.08 & 2.88 \\
\hline 5 & 50 & 6.71 & $2 . \overline{26}$ & 2.64 & 3.12 \\
\hline 5 & 75 & 7.77 & 2.90 & 3.28 & 3.76 \\
\hline \multicolumn{6}{|c|}{ Diversity Gains (dB) at $P_{e}=10^{-3}$} \\
\hline \multirow{2}{*}{$\begin{array}{c}\text { Data Rate } \\
\text { (kbps) }\end{array}$} & \multirow{2}{*}{$\begin{array}{l}\text { Mobile Speed } \\
\text { (miles/hr) }\end{array}$} & \multicolumn{2}{|c|}{ 1 Path } & \multicolumn{2}{|c|}{2 Paths } \\
\hline & & $L=15$ & $L=511$ & $L=15$ & $L=511$ \\
\hline 10 & 50 & 0 & 6.26 & 10.1 & 11.22 \\
\hline 5 & 50 & 0 & 8.15 & 9.07 & 11.31 \\
\hline 5 & 75 & 0 & 9.46 & 9.8 & 11.64 \\
\hline \multicolumn{6}{|c|}{ Total Gains (dB) at $P_{e}=10^{-3}$} \\
\hline \multirow{2}{*}{$\begin{array}{c}\text { Data Rate } \\
\text { (kbps) }\end{array}$} & \multirow{2}{*}{$\begin{array}{l}\text { Mobile Speed } \\
\text { (miles/hr) }\end{array}$} & \multicolumn{2}{|c|}{ 1 Path } & \multicolumn{2}{|c|}{2 Paths } \\
\hline & & $L=15$ & $L=511$ & $L=15$ & $L=511$ \\
\hline 10 & 50 & 5.30 & 8.02 & 12.18 & 14.10 \\
\hline 5 & 50 & 6.71 & 10.41 & 11.71 & 14.43 \\
\hline 5 & 75 & 7.77 & 12.36 & 13.08 & 15.40 \\
\hline
\end{tabular}

The coding gain that can be achieved on an AWGN channel by this code is bounded by the following inequality [1]

$$
\text { coding gain } \leq 10 \log _{10}\left(r d_{\text {free }}\right) \text {. }
$$

For the chosen code, $r=1 / 2$ and $d_{\text {free }}=5$ and the upper bound is $3.98 \mathrm{~dB}$. However, the actual coding gain on non-AWGN channels may surpass this bound [28].

The coded systems are simulated for channels with one and two paths for different mobile speeds and data rates. The diversity and coding gains are summarized in Table I for $P_{e}=10^{-3}$. Each row in the table corresponds to a different combination of mobile speed and data rate. The following conclusions can be drawn from the simulation results summarized in Table I and Fig. 9.

1) The coded time-selective signaling system exploiting joint multipath-Doppler diversity outperforms a coded traditional RAKE receiver exploiting only multipath diversity (seen earlier for an uncoded system). This is illustrated in Fig. 9.

2) The system with no diversity, that is, a single path system with $L=15$, has the highest coding gain. However, the total (diversity plus coding) gain for the systems with diversity is always greater than the total (coding) gain in the absence of diversity.

3) Some of the coding gain that is lost by the addition of the first diversity component is regained by adding more diversity components. We see that in the two-path case, which is more realistic, in addition to the diversity gain from time-spreading, there is also an increase in the coding gain compared to the system with only multipath diversity. For example, the coding gain for a multipath diversity system $(L=15)$ at a data rate of $5 \mathrm{~kb} / \mathrm{s}$ and a mobile speed of $50 \mathrm{mi} / \mathrm{h}$ is $2.64 \mathrm{~dB}$. However, the coding gain for the time-selective signaling system with joint multipath-Doppler diversity $(L=511)$ under the same channel conditions is $3.12 \mathrm{~dB}$. For a high level of diversity, we expect the coding gain to be similar to that

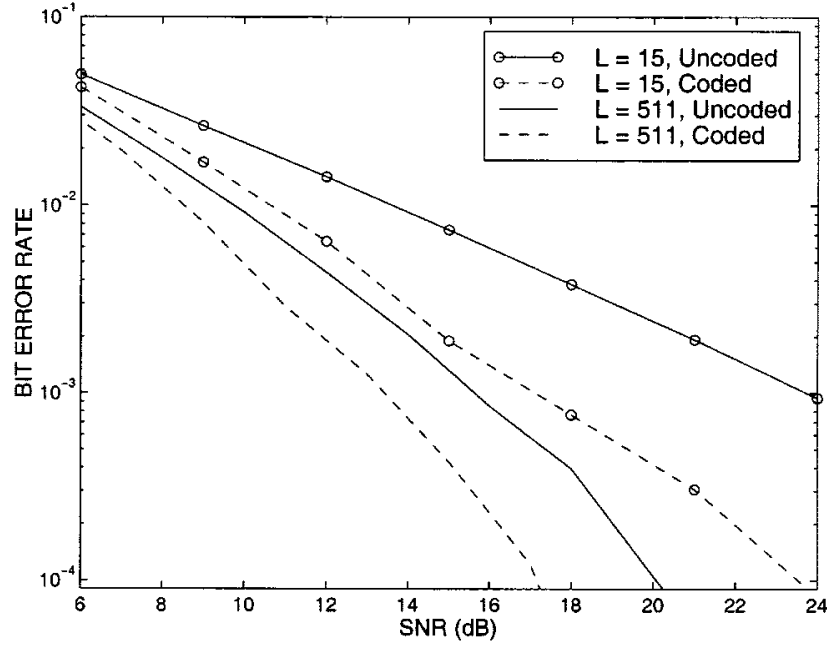

Fig. 9. Performance comparison of coded system with and without diversity-BER versus SNR per symbol-uncoded bit rate $=5 \mathrm{~kb} / \mathrm{s}$, intersymbol duration $=T / T_{c}=15$, single path, mobile speed $=50 \mathrm{mi} / \mathrm{h}$, length of spreading code $=L$

of the coding gain for an AWGN channel (bounded by $3.98 \mathrm{~dB}$ in this case).

4) For a fixed signaling duration, the coding gain increases with the mobile speed. This can be attributed to the fact that the code is able to see more variations of the channel within its constraint length and is therefore able to exploit time-diversity.

These results emphasize the importance of exploiting joint multipath-Doppler diversity to bring the fading channel closer to an AWGN channel, thereby enhancing the performance of simple codes. It is advantageous to keep the constraint length small to keep the complexity low, while maximally exploiting diversity via time-selective signaling and reception.

\section{B. Comparison with a Block Interleaving Scheme}

A commonly used technique to improve the efficacy of coding techniques over fading channels is interleaving [29]. In interleaving, coded input bits are reordered before transmission and put back in the original order before decoding. This is done to achieve time-variability of the fading process within the constraint length of the code and thereby achieve independent fading in successive bits. It is worth noting that interleaving does not improve performance without coding. On the other hand, the proposed time-selective scheme provides performance improvements even in an uncoded system.

In this section, the proposed time-selective signaling technique is compared with a block interleaving scheme via simulations. Both time-selective signaling and block interleaving incur a delay, and the two systems are compared for comparable delays. The delay for a time-selective signaling scheme is equal to the ratio $T_{s} / T$ for one-shot detection. The delay involved in an $M \times M$ block interleaver-deinterleaver combination is $2 M^{2}$. Thus, in the comparison, $T_{s} / T$ is chosen approximately equal to $2 M^{2}$.

Fig. 10 shows the simulation results for a channel with two paths and a mobile speed of $50 \mathrm{mi} / \mathrm{h}$. The time-selective 


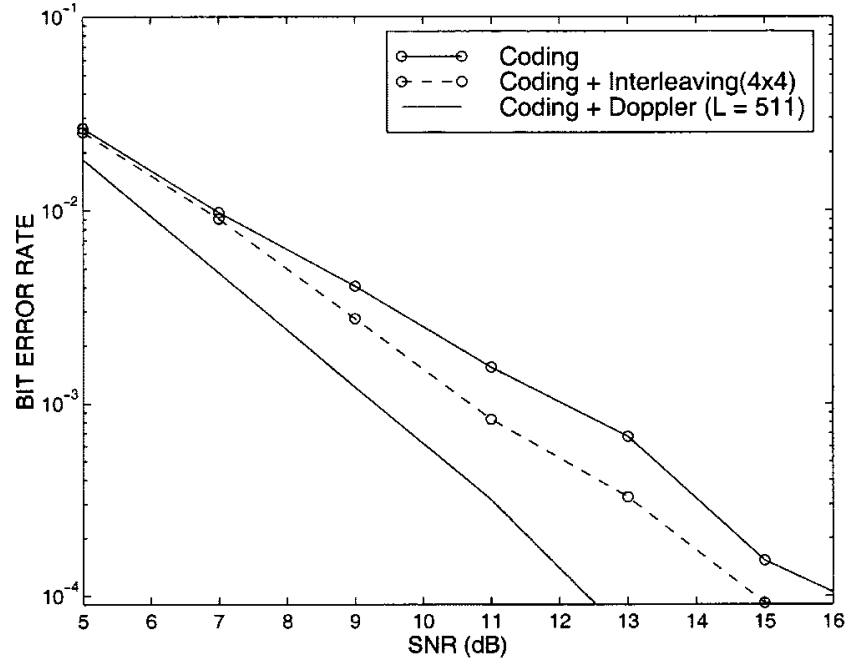

Fig. 10. Comparison of time-selective signaling scheme with block interleaving-BER versus SNR per symbol-mobile speed $=50 \mathrm{mi} / \mathrm{h}$, length of spreading code $=L$, intersymbol duration $=T / T_{c}=15$, two paths, ratio of average energy of the two paths $=2: 1$.

signaling scheme employs a length-511 spreading code, corresponding to a delay of approximately 34 bits (intersymbol duration $=15$ chips). The block interleaving scheme uses an $M=4$ block corresponding to a delay of 32 bits. As evident, the coded time-selective scheme provides substantially better performance compared to coded block interleaving.

Recall that asymptotically, with increasing delay, the coded time-selective signaling scheme can potentially approach the performance achieved on an AWGN channel. However, bit interleaving does not have similar asymptotic performance-a good approximation is the performance of a diversity system with number of diversity components equal to the minimum free distance of the convolutional code [1].

\section{CONCLUSIONS}

Time-selective signaling and reception techniques developed in this paper can significantly improve the performance of CDMA systems in multipath fading channels. While the RAKE receiver in existing CDMA systems exploits only multipath diversity, our approach is based on a time-frequency generalization of the RAKE receiver that maximally exploits channel diversity via joint multipath-Doppler processing. Joint multipath-Doppler diversity is facilitated by a signaling scheme that spreads the spread-spectrum symbol waveform in time to make the channel time-selective. Analytical and simulated results show compelling gains due to time-selective signaling and reception for realistic single path and multipath fading scenarios. Moreover, our results show that the effects of the ISI introduced by the overlapping temporally spread codes are relatively negligible in the single-user case. This can be attributed to the excellent correlation properties of $m$-sequences, which improve with increasing code length. This suggests that the one-shot detector that ignores ISI is sufficient in most practical situations. Furthermore, the one-shot detector can be efficiently implemented in practice using a bank of conventional RAKE receivers.
Practical implementation of the proposed system raises some other issues that are worth mentioning. Even though the system does not require additional bandwidth, matched filter correlation is performed over longer durations that may raise some dynamic range issues. Moreover, the presence of overlapping codes also increases the dynamic range at the receiver. However, since the symbol energy is kept constant, the maximum signal strength at the receiver increases only as the square root of the number of overlapping symbols.

The substantially higher level of joint multipath-Doppler diversity brings the fading channel closer to an AWGN channel, thereby facilitating the use of simple existing coding techniques. The use of simple codes helps to reduce the decoding complexity at the receiver. Simulation results for a coded system show that the additional diversity exploited by the time-selective signaling and reception scheme can enhance the performance of the code in addition to providing the diversity gain. The proposed scheme also provides a powerful alternative to interleaving and delivers substantially improved performance. Moreover, unlike interleaving, the time-selective signaling and reception schemes provide improved performance even in the absence of coding.

Finally a few words about possible extensions. More extensions to multiuser scenarios are possible based on the results in [21] and [26]. Since we assumed perfect channel estimates for maximal-ratio combining, an important practical consideration that needs to be studied is the effect of channel estimation errors on performance. In essence, the transceivers proposed in this paper suffer from the same channel estimation issues as conventional RAKE receivers but offer an inherently higher level of diversity. In this context, other linear diversity combining techniques [30], which do not require channel estimation, could be useful alternatives. Extensions to orthogonal signaling and noncoherent reception also warrant further investigation. Finally, it would also be interesting to compare the proposed time-selective signaling scheme with other interleaving schemes that are more efficient than block interleaving [31], [32].

\section{APPENDIX}

Proof: We know that $n(t)$ is a zero-mean white complex Gaussian process with power spectral density $\mathcal{N}_{0}$. Also, $n(t)$ is independent of $\left\{h_{n, k}^{i}\right\}$. Therefore

$$
\begin{aligned}
E\left[N^{i}\right] & =0 \text { and } \operatorname{var}\left[N^{i}\right] \\
& =\sum_{n=0}^{N} \sum_{k=-K}^{K} E\left[\left|h_{n, k}^{i}\right|^{2}\right] \mathcal{N}_{0}=C \mathcal{N}_{0}
\end{aligned}
$$

where we have used (26) in the second relation. The Lindeberg condition is a sufficient condition for the CLT. Applying CLT to $N^{i}$ [27], we get

$$
N^{i} \rightarrow N\left(0, C \mathcal{N}_{0}\right)
$$

For the signal term $S^{i}$ we have

$$
E\left[S^{i}\right]=\sqrt{E_{s}} \sum_{n=0}^{N} \sum_{k=-K}^{K} E\left[\left|h_{n, k}^{i}\right|^{2}\right]=C \sqrt{E_{s}}
$$


and

$$
\operatorname{var}\left(S^{i}\right)=E_{s} \sum_{n=0}^{N} \sum_{k=-K}^{K}\left\{E\left[\left|h_{n, k}^{i}\right|^{4}\right]-E^{2}\left[\left|h_{n, k}^{i}\right|^{2}\right]\right\} .
$$

Since $\left\{h_{n, k}^{i}\right\}$ are zero-mean complex Gaussian random variables, we have

$$
E\left[\left|h_{n, k}^{i}\right|^{4}\right]=2 E^{2}\left[\left|h_{n, k}^{i}\right|^{2}\right]
$$

and thus

$$
\operatorname{var}\left[S^{i}\right]=E_{s} \sum_{n=0}^{N} \sum_{k=-K}^{K} E^{2}\left[\left|h_{n, k}^{i}\right|^{2}\right]
$$

If (27) holds

$$
S^{i} \rightarrow E\left[S^{i}\right]=C \sqrt{E_{s}}
$$

by the law of large numbers. Combining (32) and (37), we get

$$
Z^{i} \rightarrow N\left(C \sqrt{E_{s}}, C \mathcal{N}_{0}\right)
$$

\section{REFERENCES}

[1] J. G. Proakis, Digital Communications. New York: McGraw-Hill, 1995.

[2] G. L. Turin, "Introduction to spread-spectrum antimultipath techniques and their application to urban digital radio," Proc. IEEE, vol. 68, pp. 328-353, Mar 1980.

[3] R. Price and P. E. Green, "A communication technique for multipath channels," Proc. IEEE, vol. 46, pp. 555-570, Mar 1958.

[4] J. K. Cavers, "An analysis of pilot symbol assisted modulation for Rayleigh fading channels," IEEE Trans. Veh. Technol., vol. 40, pp. 689-693, Nov. 1991.

[5] B. Sklar, "Rayleigh fading channels in mobile digital communication systems-Part II: Mitigation,” IEEE Commun. Mag., vol. 37, pp. 103-109, July 1997.

[6] A. M. Sayeed and B. Aazhang, "Exploiting Doppler diversity in mobile wireless communications," in Proc. 1997 Conf. Information Sciences and Systems (CISS'97), Baltimore, MD, 1997, pp. 287-292.

[7] — "Joint multipath-doppler diversity in mobile wireless communications," IEEE Trans. Commun., vol. 47, pp. 123-132, Jan. 1999.

[8] — - "Communication over multipath fading channels : A time-frequency perspective," in Wireless Communications: TDMA versus CDMA, S. G. Glisic and P. A. Leppanen, Eds. Norwell, MA: Kluwer, 1997, pp. 73-98.

[9] J. Ventura-Traveset, G. Caire, E. Biglieri, and G. Taricco, "Impact of diversity reception on fading channels with coded modulation-Part 1 : Coherent detection," IEEE Trans. Commun., vol. 45, pp. 563-572, May 1997.

[10] G. W. Wornell, "Spread-response precoding for communication over fading channels," IEEE Trans. Inform. Theory, vol. 42, pp. 488-501, Mar. 1996.

[11] — "Spread-signature CDMA: Efficient multiuser communication in the presence of fading," IEEE Trans. Inform. Theory, vol. 41, pp. 1418-1438, Sept. 1995.

[12] P. B. Rapajic and B. S. Vucetic, "Adaptive receiver structures for asynchronous CDMA systems," IEEE J. Select. Areas Commun., vol. 12, pp. 685-697, May 1994.

[13] P. A. Bello, "Characterization of randomly time-varying linear channels," IEEE Trans. Commun., vol. COM-11, pp. 360-393, 1963.

[14] A. M. Sayeed, "Canonical multipath-doppler coordinates in wireless communications," in Proc. 36th Annu. Allerton Conf. Communications, Control, and Computing, Monticello, IL, 1998, pp. 536-545.
[15] T. Kailath, "Measurements on time-variant communication channels," IEEE Trans. Inform. Theory, vol. IT-8, pp. S229-S236, Sept. 1962.

[16] P. A. Bello, "Measurement of random time-variant channels," IEEE Trans. Inform. Theory, vol. IT-15, pp. 469-475, July 1969.

[17] T. S. Rappaport, Wireless Communications: Principles and Practice. Englewood Cliffs, NJ: Prentice-Hall, 1995.

[18] G. Ungerboeck, "Adaptive maximum-likelihood receiver for carrier-modulated data-transmission systems," IEEE Trans. Commun., vol. COM-22, pp. 624-636, May 1974.

[19] G. D. Forney, "Maximum likelihood sequence estimation of digital sequences in the presence of intersymbol interference," IEEE Trans. Inform. Theory, vol. IT-18, pp. 363-378, May 1972.

[20] B. D. Hart and D. P. Taylor, "Extended MLSE diversity receiver for the time- and frequency-selective channel," IEEE Trans. Commun., vol. 45, pp. 322-333, Mar. 1997.

[21] A. M. Sayeed, A. Sendonaris, and B. Aazhang, "Multiuser detection in fast fading multipath environments," in Proc. 31st Asilomar Conf. Signals, Systems, and Computers, Nov 1997.

[22] Z. Zvonar and D. Brady, "Linear multipath-decorrelating receivers for CDMA frequency-selective fading channels," IEEE Trans. Commun., vol. 44, pp. 650-653, June 1996.

[23] R. Lupas and S. Verdu, "Linear multiuser detectors for synchronous code-division multiple-access channels," IEEE Trans. Inform. Theory, vol. 35, pp. 123-136, Jan 1989.

[24] M. K. Varanasi and B. Aazhang, "Multistage detection in asynchronous code-division multiple-access communications," IEEE Trans. Commun., vol. 38, pp. 509-519, Apr. 1990.

[25] R. Lupas and S. Verdu, "Near-far resistance of multiuser detectors in asynchronous channels," IEEE Trans. Commun., vol. 38, pp. 496-508, Apr. 1990.

[26] A. M. Sayeed, A. Sendonaris, and B. Aazhang, "Multiuser detection in fast fading multipath environments," IEEE J. Select. Areas Commun., vol. 16, pp. 1691-1701, Dec. 1998.

[27] P. Billingsley, Probability and Measure. New York: Wiley, 1979.

[28] I. M. Jacobs, "Practical applications of coding," IEEE Trans. Inform. Theory, vol. IT-20, pp. 305-310, May 1974.

[29] A. J. Viterbi, CDMA : Principles of Spread Spectrum Communication. Reading, MA: Addison-Wesley, 1995.

[30] D. G. Brennan, "Linear diversity combining techniques," in Proc. IEEE, vol. 47, June 1959, pp. 1075-1102.

[31] J. L. Ramsey, "Realization of optimum interleavers," IEEE Trans. Inform. Theory, vol. IT-16, pp. 338-345, May 1970.

[32] G. D. Forney, "Burst-correcting codes for the classic bursty channel," IEEE Trans. Commun., vol. COM-19, pp. 772-781, Oct. 1971.

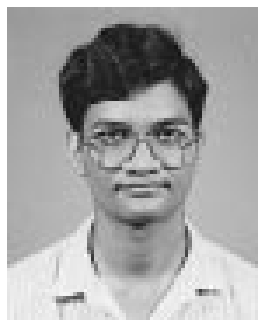

Srikrishna Bhashyam (S’96) received the B.Tech. degree in electronics and communication engineering from the Indian Institute of Technology, Madras, India, and the M.S. degree in electrical engineering from Rice University, Houston, TX, in 1996 and 1998, respectively. He is currently working toward the Ph.D. degree at Rice University.

His research interests include wireless multimedia communications and information theory.

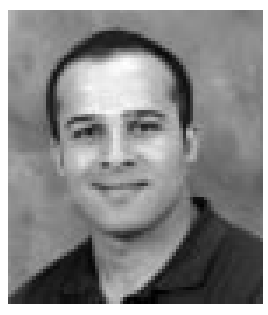

Akbar M. Sayeed (S'87-M'97) received the B.S. degree from the University of Wisconsin at Madison, in 1991, and the M.S. and Ph.D. degrees, in 1993 an 1996, respectively, from the University of Illinois at Urbana-Champaign, all in electrical engineering.

While at the University of Illinois, he was a Research Assistant in the Coordinated Science Laboratory, and from 1992 to 1995 , he was the Schlumberger Fellow in signal processing. From 1996 to 1997, he was a Postdoctoral Fellow at Rice University. Since August 1997, he has been with the University of Wisconsin at Madison, where he is currently an Assistant Professor in Electrical and Computer Engineering. His research interests include wireless communications, statistical signal processing, and time-frequency and wavelet analysis.

Dr. Sayeed received the National Science Foundation CAREER Award in 1999. 


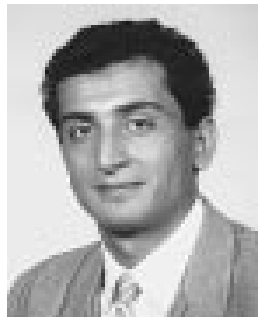

Behnaam Aazhang (S'82-M'85-SM'91-F'99) received the B.S. (with highest honors), M.S., and $\mathrm{Ph} . \mathrm{D}$. degrees in electrical and computer engineering from the University of Illinois at Urbana-Champaign in 1981, 1983, and 1986, respectively.

From 1981 to 1985, he was a Research Assistant in the Coordinated Science Laboratory at the University of Illinois. In August 1985, he joined the faculty of Rice University, Houston, TX, where he is now a Professor in the Department of Electrical and Computer Engineering and the Director of the Center for Multimedia Communications. He has been a Visiting Professor at the IBM Federal Systems Company in Houston, TX, the Laboratory for Communication Technology at the Swiss Federal Institute of Technology (ETH) in Zurich, Switzerland, the Telecommunications Laboratory at the University of Oulu, Oulu, Finland, and at the US Air Force Phillips Laboratory, Albuquerque, NM. His research interests are in the areas of communication theory, information theory, and their applications with emphasis on multiple-access communications, cellular mobile radio communications, and optical communication networks.

Dr. Aazhang is a recipient of the 1993 Alcoa Foundation Award, the 1987-1989 NSF Engineering Initiation Award, and the 1984-1985 IBM Graduate Fellowship. He is a member of Tau Beta Pi and Eta Kappa Nu. $\mathrm{He}$ has served as the Editor for Spread Spectrum Networks of the IEEE TRANSACTIONS ON COMMUNICATIONS from 1993 to 1998, the Treasurer of the IEEE Information Theory Society from 1995 to 1998, the Technical Area Chair of the 1997 Asilomar Conference, the Secretary of the Information Theory Society from 1990 to 1993, and the Publications Chairman of the 1993 IEEE International Symposium on Information Theory. 